\title{
Erratum to: Oligoremora rhenana n. g. n. sp., a new echeneid fish (Percomorpha, Echeneoidei) from the Oligocene of the Grube Unterfeld ("Frauenweiler") clay pit
}

\author{
Norbert Micklich $^{1}$ - Růžena Gregorová ${ }^{2}$ Alexandre F. Bannikov ${ }^{3}$. \\ Dorin-Sorin Baciu ${ }^{4} \cdot$ Ionuţ Grădianu $^{5}$ - Giorgio Carnevale ${ }^{6}$
}

Published online: 12 September 2016

(C) Paläontologische Gesellschaft 2016

\section{Erratum to: Paläontol Z \\ DOI 10.1007/s12542-016-0303-y}

In the original article the keywords contained printing errors. The correct "Keywords" and "Schlüsselwörter" are reproduced in full below. We apologize for this error.

Keywords Morphology · Taphonomy · Palaeoecology · Echeneidae - Lower Oligocene - Rauenberg - S Germany · Eastern Paratethys

Schlüsselwörter Morphologie · Taphonomie .

Paläoökologie · Echeneidae · Unter-Oligozän · Rauenberg · Süddeutschland · Östliche Paratethys

The online version of the original article can be found under doi:10.1007/s12542-016-0303-y.

Norbert Micklich

micklich@hlmd.de

Růžena Gregorová

rgregorova@mzm.cz

Alexandre F. Bannikov

aban@paleo.ru

Dorin-Sorin Baciu

dsbaciu@gmail.com

Ionuţ Grădianu

igradianu@hotmail.com

Giorgio Carnevale

giorgio.carnevale@unito.it

1 Natural History Department, Hessisches Landesmuseum Darmstadt, Friedensplatz 1, 64283 Darmstadt, Germany
2 Department of Geology and Paleontology, Moravian Museum Brno, Zelny trh 6, 65937 Brno, Czech Republic

3 Borisyak Paleontological Institute, Russian Academy of Sciences, Profsoyuznaya 123, Moscow 117997, Russia

4 Department of Geology, “Al. I. Cuza” University of Iaşi (Romania), Bd. Carol I, 20A, 700505 Iaşi, Romania

5 Natural Sciences Museum, Str. Petru Rares 26, 610119 Piatra Neamţ, Romania

6 Dipartimento di Scienze della Terra, Università degli Studi di Torino, Via Valperga Caluso 35, 10125 Turin, Italy 